\title{
Survival analysis of advanced lung cancer patients undergoing personalised treatment or chemotherapy in a real clinic
}

\author{
Justyna Błach ${ }^{1,2}$, Paweł Krawczyk ${ }^{1}$, Juliusz Pankowski ${ }^{3}$, Jarosław Buczkowski ${ }^{1,4}$, \\ Izabela Chmielewska ${ }^{1}$, Małgorzata Frą $k^{1}$, Artur Szlubowski ${ }^{3,5}$, Piotr Krudys ${ }^{5}$, Robert Kieszko ${ }^{1}$, \\ Kamila Wojas-Krawczyk ${ }^{1}$, Katarzyna Reszka ${ }^{6}$, Tomek Kucharczyk ${ }^{1}$, Janusz Milanowski ${ }^{1}$
}

\begin{abstract}
${ }^{1}$ Department of Pneumonology, Oncology, and Allergology, Medical University of Lublin, Lublin, Poland

${ }^{2}$ Department of Clinical Immunology, Medical University of Lublin, Lublin, Poland ${ }^{3}$ Specialist Hospital for Lung Diseases “Rebirth”, Klara Jelska, Poland

${ }^{4}$ Department of Lung Diseases and Intensive Care, Independent Public Provincial John of God Hospital, Lublin, Poland

5Izerski Pulmonology and Chemotherapy Center "IZER-MED”, Szklarska Poręba, Poland

${ }^{6}$ Genetics and Immunology Institute GENIM LCC, Lublin, Poland
\end{abstract}

Submitted: 17 August 2020

Accepted: 12 November 2020

Arch Med Sci

DOI: https://doi.org/10.5114/aoms/130375

Copyright (c) 2020 Termedia \& Banach

\section{Abstract}

Introduction: The importance of modern treatments for the extension of overall survival in advanced lung cancer (LC) patients is rarely reported in clinical trials (crossover effect). Recent clinical trials have compared experimental treatment methods and shown that chemotherapy is no longer a comparator. We studied the relevance of innovative treatment to the extension of overall survival in Polish lung cancer patients.

Material and methods: We described the outcome in 1463 patients diagnosed and treated for advanced LC. The study included patients receiving all available forms of treatment, i.e. chemotherapy, immunotherapy, EGFR tyrosine kinase inhibitors, ALK inhibitors, and best supportive care (BSC). Results: Median OS (mOS) for the whole group of patients was 6.5 months. mOS was significantly higher in patients with SCC (8.0 months) and AC (7.0 months) compared to patients with SCLC (6 months) and NSCLC NOS (3.5 months). mOS was 30 months for EGFR TKI-treated patients, 34 months for patients receiving second-line immunotherapy, 8.5 months for chemotherapy patients, and 1.0 month for patients who received BSC. mOS for patients treated with ALK inhibitors and first-line immunotherapy was not reached. The use of targeted therapies or immunotherapies significantly $(p<0.0001)$ reduced the risk of death compared to chemotherapy $(\mathrm{HR}=$ $0.373,95 \% \mathrm{Cl}: 0.288-0.484$ and $\mathrm{HR}=0.313,95 \% \mathrm{Cl}: 0.255-0.385)$.

Conclusions: The use of modern therapies in one of the treatment lines compared to chemotherapy significantly increased the long-term survival of advanced LC patients (34.5 vs. 8.5 months, $\mathrm{HR}=0.336,95 \% \mathrm{Cl}: 0.284-$ $0.397, p<0.0001)$. Correct and early LC diagnosis is required, because patients with late diagnosis have a particularly poor prognosis.

Key words: lung cancer, chemotherapy, molecularly targeted therapy, immunotherapy, overall survival.
Corresponding author: Justyna Błach MD Department of Pneumonology, Oncology and Allergology Department of Clinical Immunology Medical University of Lublin, Poland Poland E-mail: justynablach91@ gmail.com 


\section{Introduction}

Lung cancer remains a significant clinical problem and is the primary cause of death due to malignancies. Most patients are diagnosed at an advanced stage of the disease. Non-small cell lung cancer (NSCLC) accounts for $80-85 \%$ of the diagnosed cases, of which the predominant subtypes are adenocarcinoma (AC, $40-50 \%$ of NSCLC) and squamous cell carcinoma (SCC, 20-30\% of NSCLC) [1, 2].

Treatment options for advanced non-small cell lung cancer patients have substantially broadened in the past few years. Understanding the importance of genetic alterations, like activating the epidermal growth factor receptor gene (EGFR) mutations, the anaplastic lymphoma kinase gene $(A L K)$, and the c-ros oncogene 1 gene (ROS1) rearrangement, has led to the development of effective molecularly targeted therapies. Tests for these alterations have been contained into standard diagnostic algorithms. Nowadays, molecularly targeted treatments of patients with molecularly altered tumours could be used very successfully in advanced stages of NSCLC. The type of genetic alterations is related to gender, smoking status, and the histological type of NSCLC [3].

Druggable mutations in the EGFR gene occur in exons 18-21, which encode the tyrosine kinase domain of EGFR. These cause autonomous activation of EGFR and excessive proliferation of cancer cells. EGFR gene mutations are more common in females, in non-smokers, and in adenocarcinoma patients [4]. Epidermal growth factor receptor gene mutations are detected in $10 \%$ of Caucasian NSCLC patients [5]. Rearrangements in the anaplastic lymphoma kinase gene and the ROS1 gene appear in 5\% and 1\% of NSCLC patients, almost exclusively in adenocarcinoma patients [6]. The other druggable genetic alterations in NSCLC patients are the BRAF gene mutation and the NTRK1-3 (neurotrophic tyrosine receptor kinase) genes rearrangement. These abnormalities occur with a frequency of $1 \%$ of NSCLC patients. However, many therapies for NSCLC patients with exceedingly rare genetic abnormalities remain in clinical trials (e.g. RET and MET inhibitors).

EGFR and ALK tyrosine kinase inhibitors (TKIS) have become the standard of first-line treatment in NSCLC. In Poland, erlotinib and gefitinib are reimbursed in the first and second line of treatment, afatinib in the first line of treatment, and osimertinib in patients with disease progression after treatment with erlotinib, gefitinib, or afatinib. In patients with the ALK gene rearrangement, ALK inhibitors of the first (crizotinib), the second (alectinib, ceritinib and brigatinib), and the third generation (lorlatinib) proved effectiveness in NSCLC therapy. In Poland, only crizotinib, alectinib, and ceritinib are reimbursed in the first and further lines of treatment in NSCLC patients.

Immunotherapies used in lung cancer patients employ the anti-PD-1 (programmed death 1) and anti-PD-L1 (programmed death ligand 1) monoclonal antibodies. Inhibitors of immune checkpoints release cytotoxic T cell (CTLS) function by blocking the inhibitory signals transmitted from the binding of PD-L1 on tumour cells to PD-1 on CTLs [7]. An analysis of three global clinical trials involving 4784 patients with NSCLC found that $68 \%$ of patients with advanced NSCLC had measurable PDL1 (on $\geq 1 \%$ of tumour cells) and $28 \%$ of patients had PD-L1 on $\geq 50 \%$ of tumour cells. When separated by histology, $74 \%$ of patients with nonsquamous carcinoma and $81 \%$ of patients with SCC had measurable PD-L1 expression [8].

Monoclonal antibodies anti-PD-1 (nivolumab, pembrolizumab) and anti-PD-L1 (atezolizumab, durvalumab, and avelumab) are used in NSCLC therapy. In the European Union, nivolumab and atezolizumab have been registered for the second line of treatment in NSCLC patients, regardless of PD-L1 expression on cancer cells. In contrast, pembrolizumab can be used in the first line of treatment in patients with PD-L1 expression on $\geq 50 \%$ of cancer cells, or in the second line of treatment in patients with PD-L1 expression on $\geq 1 \%$ of cancer cells. Pembrolizumab could be also used in combination with first-line chemotherapy in NSCLC patients, regardless of PD-L1 expression on cancer cells. Moreover, durvalumab is used as maintenance therapy after concurrent chemoradiotherapy in locally advanced patients with NSCLC. Furthermore, atezolizumab and durvalum$a b$ in combination with first-line chemotherapy have found application in the treatment of small cell lung cancer (SCLC) [9]. Unfortunately, in Poland only pembrolizumab in the first treatment line in NSCLC patients and atezolizumab in the second treatment line in NSCLC patients as well as nivolumab in the second treatment line in SCC patients are reimbursed.

Despite these limitations in the availability of personalised therapies and because of the still widespread use of chemotherapy in Poland, we have decided to demonstrate the relevance of innovation treatment to the extension of overall survival in Polish lung cancer patients.

\section{Material and methods}

\section{Patient enrolment and data collection}

A total of 1463 lung cancer (LC) patients were retrospectively enrolled in the study ( 975 men and 488 women, with median age of 65 years). These patients were diagnosed and treated for advanced LC in 2016-2018 in 3 Polish lung cancer centres. 
Diagnosis of LC was based on the evaluation of histological and cytological specimens. Methods of sample collection were endobronchial forceps biopsies, transbronchial biopsies, and transoesophageal biopsies (endobronchial ultrasound (EBUS) transbronchial needle aspiration (TBNA) and endoscopic ultrasound (EUS) fine needle aspiration (FNA)).

The collected data included demographic factors, LC histopathological diagnosis, results of assessment of the molecular predictive factors qualifying the patients for molecularly targeted treatment (EGFR gene mutations, ALK gene rearrangement), assessment of PD-L1 expression on cancer cells, treatment regimens (including EGFR TKIS, ALK TKIs immunotherapy and chemotherapy), and overall survival (OS, Table I).

The use of several chemotherapy lines in patients receiving chemotherapy and the use of subsequent chemotherapy in patients receiving personalised treatment were allowed. Treatment was standard and complied with the recommendations of the ESMO Clinical Practice Guidelines on metastatic non-small cell lung cancer (NSCLC) [10]. The treatment regimen was not modified by any individual doctor. EGFR TKIS, ALK TKIs, and immunotherapy were used in accordance with the guidelines of the Polish Drug Program of the
National Health Fund and in accordance with the Summary of Product Characteristics (SmPC). Chemotherapy regimens were used in accordance with the Summary of Product Characteristics for individual cytostatics.

\section{EGFR gene mutations analysis}

DNA was extracted from tumour tissue or tumour cells obtained during routine diagnostic or therapeutic procedures. Formalin-fixed paraffin-embedded (FFPE) materials or cytological slides containing at least $10 \%$ of tumour cells were used for molecular examination. Mutations of the EGFR gene (NM_005228.4) were tested using routine real-time PCR procedures and the EntroGen EGFR Mutations Analysis kit (USA). The mutations in exons 18 to 21 were examined.

\section{$A L K$ gene rearrangement testing}

ALK protein IHC (immunohistochemistry) staining was carried out on Ventana Benchmark GX equipment, using CE-IVD-approved anti-ALK Rabbit Monoclonal Primary Antibody (clone D5F3), utilising OptiView Amplification Kit and OptiView DAB IHC Detection Kit as a detection system. Counterstaining, using haematoxylin (Ventana Medical System, Tucson, AZ, USA), was included in

Table I. Characteristics of analysed patients, one-year survival, and overall survival in accordance with clinical factors

\begin{tabular}{|c|c|c|c|c|c|c|}
\hline \multirow[t]{2}{*}{ Feature } & \multirow[t]{2}{*}{ Total } & \multicolumn{2}{|c|}{ One-year survival, $n$ (\%) } & \multirow[t]{2}{*}{$P, \chi^{2}$} & \multirow{2}{*}{$\begin{array}{c}\text { mOS } \\
\text { [months] }\end{array}$} & \multirow[t]{2}{*}{$\mathrm{HR}, p, \chi^{2}, 95 \% \mathrm{Cl}$} \\
\hline & & Yes & No & & & \\
\hline \multicolumn{7}{|l|}{ Gender: } \\
\hline Male & 975 & $666(68.3)$ & $309(31.7)$ & \multirow{2}{*}{$\begin{array}{c}p=0.093 \\
\chi^{2}=2.807\end{array}$} & 6.0 & \multirow{2}{*}{$\begin{array}{c}\mathrm{HR}=0.8447 \\
p=0.0065 \\
\chi^{2}=7.416 \\
95 \% \mathrm{Cl}: 0.748-0.9538\end{array}$} \\
\hline Female & 488 & $312(63.9)$ & $176(36.1)$ & & 7.5 & \\
\hline \multicolumn{7}{|l|}{ Age: } \\
\hline$<65$ & 532 & $334(62.8)$ & $198(37.2)$ & \multirow{2}{*}{$\begin{array}{l}p=0.012 \\
\chi^{2}=6.24\end{array}$} & 8.0 & \multirow{2}{*}{$\begin{array}{c}\mathrm{HR}=0.8075 \\
p=0.0004 \\
\chi^{2}=12.4015 \\
95 \% \mathrm{Cl}: 0.7169-0.9095\end{array}$} \\
\hline$\geq 65$ & 931 & $644(69.2)$ & $287(30.8)$ & & 5.5 & \\
\hline \multicolumn{7}{|c|}{ Pathomorphological diagnosis: } \\
\hline SCC & 469 & $289(61.6)$ & $180(38.4)$ & \multirow{4}{*}{$\begin{array}{c}p=0.000092 \\
\chi^{2}=21.264\end{array}$} & 8.0 & \multirow{4}{*}{$\begin{array}{c}p<0.0001 \\
\chi^{2}=37.216\end{array}$} \\
\hline $\mathrm{AC}$ & 469 & $299(63.8)$ & $170(36.2)$ & & 7.0 & \\
\hline NSCLC-NOS & 175 & $133(76.0)$ & $42(24.0)$ & & 3.5 & \\
\hline SCLC & 350 & $257(73.4)$ & $93(26.6 \%)$ & & 6.0 & \\
\hline \multicolumn{7}{|l|}{ Treatment: } \\
\hline Chemo-therapy & 997 & $615(61.7)$ & $382(38.3)$ & \multirow{3}{*}{$\begin{array}{c}p<0.000001 \\
\chi^{2}=77.35\end{array}$} & 8.5 & \multirow{3}{*}{$\begin{array}{c}p<0.0001 \\
\chi^{2}=1301.3128\end{array}$} \\
\hline IKTs & 41 & $9(21.9)$ & $32(78.1)$ & & 34.5 & \\
\hline Immuno-therapy & 72 & $12(16.7)$ & $60(83.3)$ & & 28.5 & \\
\hline
\end{tabular}


the staining protocol. Rabbit monoclonal negative control immunoglobulin (Ventana Medical System, Tucson, AZ, USA) was used as a negative control. After staining, all glass slides were washed and dehydrated in a series of two $96 \%$ ethanol and two xylene washing steps, and then cover-slipped. Next, the slides were assessed by two pathologists using an Olympus BX41 microscope.

All positive results of abnormal ALK protein expression obtained through the IHC method were re-evaluated using the FISH (fluorescence in situ hybridisation) method to visualise the presence of the ALK gene (NM_004304.3) rearrangement using the Vysis ALK Break Apart FISH Probe Kit (Abbot Molecular, USA), Paraffin-Pretreatment IV and Post-Hybridization Wash Buffer Kit (Abbot Molecular, USA), and fluorescence microscope (Nikon Eclipse 55i, Japan). The localisation and content of tumour cells in the specimens were examined with H\&E staining in serially prepared slides. The manner of interpretation of the FISH results was in accordance with the International Association for the Study of Lung Cancer (IASLC).

\section{Examination of PD-L1 expression on tumour cells}

The analysis of the PD-L1 protein expression on tumour cells was performed on FFPE or cellblock materials cut into 3- $\mu \mathrm{m}$ sections. They were put on Thermo Scientific Superfrost Plus glass slides and preheated to $59^{\circ} \mathrm{C}$ on a hotplate for at least $3 \mathrm{~h}$. The Ventana SP263 antibody was used for PD-L1 protein IHC staining. The procedure was carried out on Ventana Benchmark GX equipment. OptiView Amplification Kit and OptiView DAB IHC Detection Kit were used as a detection system. Counterstaining using haematoxylin II (Ventana Medical System, Tucson, AZ, USA) was included in the staining protocol. Rabbit monoclonal negative control immunoglobulin (Ventana Medical System, Tucson, AZ, USA) was used as a negative control. After staining, all glass slides were washed and dehydrated in a series of two $96 \%$ ethanol and two xylene washing steps, and then cover-slipped. Next, the slides were assessed by two pathologists using an Olympus BX41 microscope.

\section{Ethics approval statement}

The protocol of the study was approved by the Committee of Ethics and Research at the Medical University of Lublin (KE-0254/5/2018).

\section{Statistical analysis}

Kaplan-Meier survival analysis was performed using MedCalc v. 18.11.6. Associations between clinical factors and overall survival were examined using the Fisher's exact test. $P$-values below 0.05 were considered significant.

\section{Results}

\section{Histopathological diagnosis}

Small cell lung cancer was diagnosed in 350 (23.9\%) patients, squamous cell lung cancer in 469 (32.05\%) patients, adenocarcinoma in 469 (32.05\%) patients, and non-small cell lung cancer not otherwise specified (NSCLC NOS) - in 175 (12\%) patients.

\section{Occurrence of predictive factors}

A total of 41 NSCLC patients had EGFR gene mutations (8.0\% of 535 tested patients), and 23 patients had ALK gene rearrangement (6.0\% of 433 examined patients). The most common EGFR gene mutations were deletion in exon 19 (24 patients, $58.5 \%$ of patients with EGFR gene mutations) and L858R substitution in exon 21 (11 patients, $26.8 \%$ of patients with EGFR gene mutations). Four patients ( $9.8 \%$ of patients with EGFR mutations) had G719X substitution in exon 18. One patient had substitution E709X in exon 18 , and 1 patient had insertion of 9 base pairs in exon 20. Six patients developed resistance to the first or second generation of EGFR TKIs due to the occurrence of T790M mutation in exon 20 in the EGFR gene.

PD-L1 expression was evaluated only in 176 patients, due to the lack of reimbursement of pembrolizumab in the period from 2016 to 2018. PD-L1 expression on $\geq 50 \%$ of cancer cells was found in 45 (25.6\%) patients.

\section{Treatment methods}

EGFR tyrosine kinase inhibitors were received by 35 patients, of which erlotinib was received by 18 patients, gefitinib by 6 patients, and afatinib by 11 patients. Four patients who had T790M mutation were qualified for osimertinib treatment. ALK inhibitors were received by 6 patients, 5 patients were treated with crizotinib, and 1 patient - with alectinib. First-line pembrolizumab therapy was applied in 14 patients, second-line immunotherapy with atezolizumab or nivolumab in 58 patients. In total 997 patients received only chemotherapy, and 353 patients received only best supportive care (BSC).

\section{Clinical and genetic factors and overall survival}

Median OS (mOS) for the whole group of patients was 6.5 months ( $95 \% \mathrm{Cl}: 6.0-7.0)$. mOS was significantly higher in women compared to men (7.5 vs. 6.0 months, respectively, $\mathrm{HR}=0.8447$, 
$p=0.0065)$, and in younger patients compared to patients over 65 years of age (8.0 vs. 5.5 months, respectively, $\mathrm{HR}=0.8075, p=0.0004)$.

Patients with SCC (8.0 months) or AC (7.0 months) demonstrated significantly higher median overall survival compared with patients with SCLC (6 months) or NSCLC NOS (3.5 months, $p<0.0001)$.

mOS for EGFR TKI-treated patients was 30 months ( $95 \% \mathrm{Cl}: 18.0-47.0)$, for patients receiving second-line immunotherapy it was 34 months (95\% Cl: 25.0-57.0), for chemotherapy patients it was 8.5 months ( $95 \% \mathrm{Cl}$ : 8.0-9.0 months), and for patients without systemic treatment (most frequently due to poor performance status) it was 1.0 month. mOS for patients treated with ALK inhibitors and first-line immunotherapy was not reached.

The use of molecularly targeted therapies (mOS $=34.5$ months) or immunotherapies (28.5 months) significantly $(p<0.0001)$ reduced the risk of death compared to chemotherapy $(H R=0.373$, 95\% Cl: $0.288-0.484$ and $\mathrm{HR}=0.313,95 \% \mathrm{Cl}$ : $0.255-0.385$, respectively).

Molecularly targeted therapies were used only in patients with non-squamous NSCLC (40 patients with adenocarcinoma and 1 patient with NOS). Immunotherapy was used for both SCC and non-squamous NSCLC (35 patients with SCC and 37 with non-squamous NSCLC). mOS did not differ significantly $(\mathrm{HR}=0.7313,95 \% \mathrm{Cl}$ : 0.3236-1.6526, $p=0.4519)$ between SCC and
non-SCC NSCLC patients treated with immunotherapy (mOS for patients with SCC was 36.5 months and mOS for patients with non-SCC was 28.5 months). The remaining patients with NSCLC and all patients diagnosed with SCLC were treated with chemotherapy or received BSC. mOS of the patients who received chemotherapy was similar $(p=0.2682)$ to that of patients with SCC, non-SCC NSCLC, and SCLC (8.5 months vs. 7.5 months vs. 9.5 months, respectively).

The use of modern therapies in one of the treatment lines compared to chemotherapy alone significantly increased the chance of long-term survival of patients with advanced LC (34.5 vs. 8.5 months, $\mathrm{HR}=0.336,95 \% \mathrm{Cl}: 0.284-0.397, p<$ $0.0001)$. mOS for untreated patients was 1 month $(\mathrm{HR}=16.119,95 \% \mathrm{Cl}: 12.281-21.157, p<0.0001$ compared to patients treated with novel therapies).

Data are presented in Table I and in Figures 1-3.

\section{One-year survival in patients with different treatment methods}

The percentage of patients over 65 years of age living 1 year after diagnosis was significantly lower than the percentage of younger patients with 1 -year survival $\left(p=0.012, \chi^{2}=6.24\right)$. No statistically significant association between the gender and 1-year survival was found. Patients with SCLC and NOS showed significantly higher risk of oneyear mortality compared to patients with SCC and AC $\left(p=0.000092, \chi^{2}=21,264\right)$. The percentage

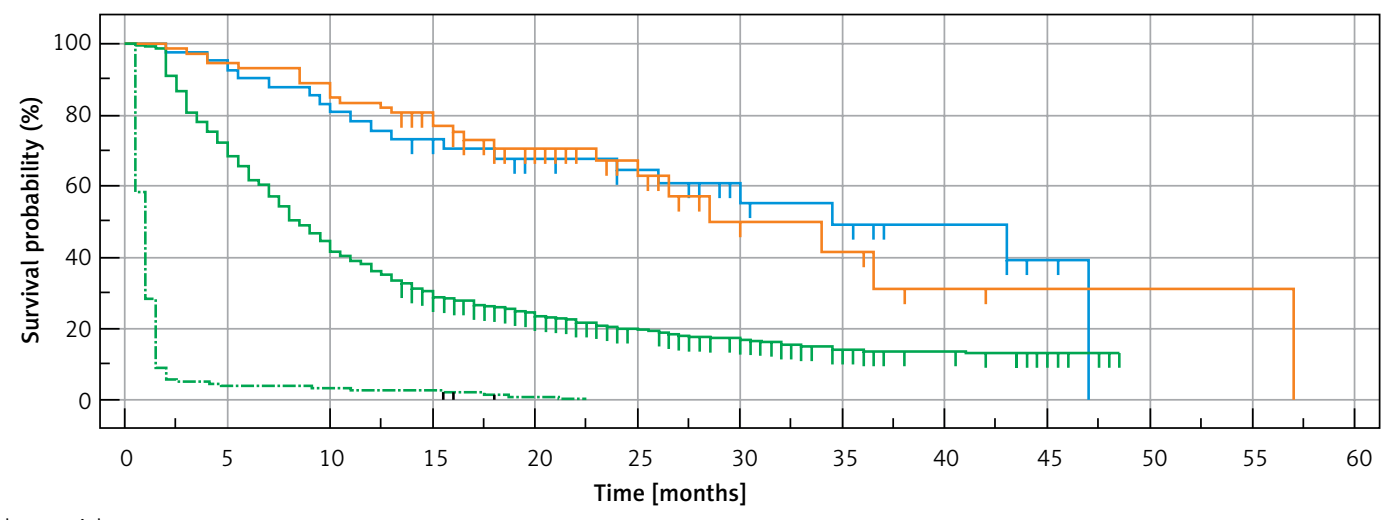

Number at risk

Group: tyrosine kinase inhibitors

\begin{tabular}{|c|c|c|c|c|c|c|c|c|c|c|c|c|}
\hline 41 & 38 & 33 & 27 & 22 & 18 & 10 & 8 & 5 & 2 & 0 & 0 & 0 \\
\hline \multicolumn{13}{|c|}{ Group: chemotherapy } \\
\hline 997 & 682 & 415 & 273 & 183 & 123 & 78 & 41 & 25 & 14 & 0 & 0 & 0 \\
\hline \multicolumn{13}{|c|}{ Group: immunotherapy } \\
\hline 72 & 68 & 61 & 40 & 24 & 14 & 6 & 5 & 2 & 1 & 1 & 1 & 0 \\
\hline \multicolumn{12}{|c|}{ Group: lack of systemic treatment } & \\
\hline 353 & 15 & 12 & 9 & 2 & 0 & 0 & 0 & 0 & 0 & 0 & 0 & 0 \\
\hline
\end{tabular}

Treatment methods

- Tyrosine kinase inhibitors - Chemotherapy - Immunotherapy --- Lack of systemic treatment

Figure 1. Survival curves of LC patients treated with tyrosine kinase inhibitors (EGFR or ALK), immunotherapies (first-line pembrolizumab and second-line atezolizumab or nivolumab), chemotherapy alone, or BSC alone (due to poor performance status) 
J. Błach, P. Krawczyk, J. Pankowski, J. Buczkowski, I. Chmielewska, M. Frąk, A. Szlubowski, P. Krudys, R. Kieszko, K. Wojas-Krawczyk, K. Reszka, T. Kucharczyk, J. Milanowski

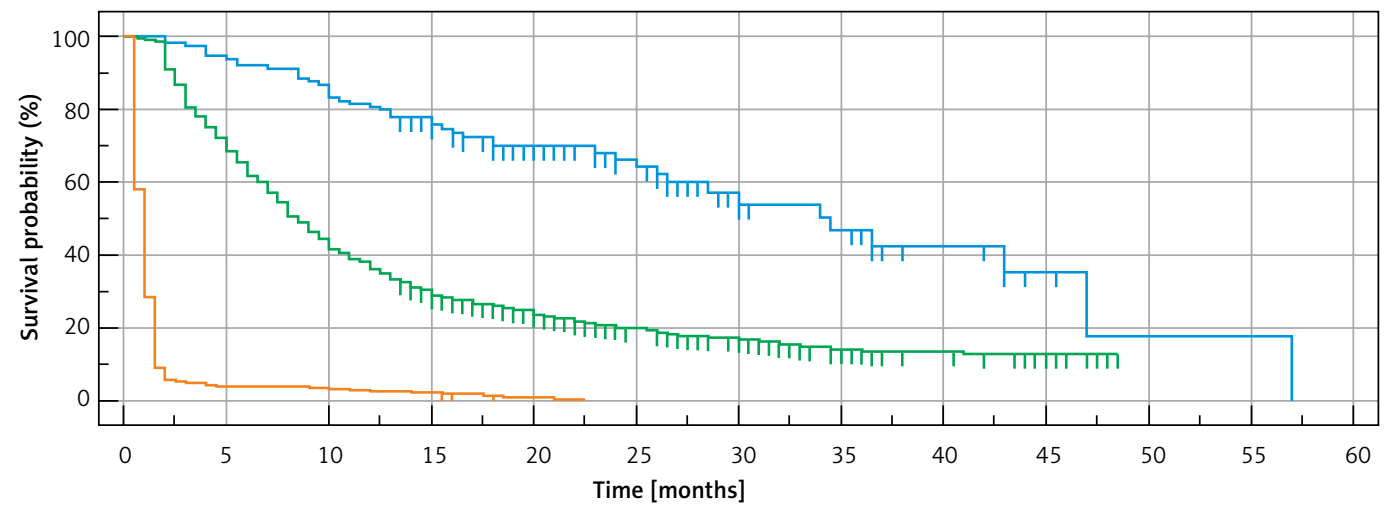

Number at risk

Group: personalised treatment

\begin{tabular}{|c|c|c|c|c|c|c|c|c|c|c|c|c|}
\hline \multicolumn{13}{|c|}{ Group: chemotherapy } \\
\hline 997 & 682 & 415 & 273 & 183 & 123 & 78 & 41 & 25 & 14 & 0 & 0 & 0 \\
\hline \multicolumn{12}{|c|}{ Group: lack of systemic treatment } & \\
\hline 353 & 15 & 12 & 9 & 2 & 0 & 0 & 0 & 0 & 0 & 0 & 0 & $c$ \\
\hline
\end{tabular}

Treatment methods

$$
\begin{array}{ccc}
- \text { Personalised treatment } & \text { Chemotherapy } \quad \text { - Lack of systemic treatment } \\
\text { T Censored data } &
\end{array}
$$

Figure 2. Survival curves of LC patients treated with personalised therapy (EGFR TKIs, ALK inhibitors, first- or second-line immunotherapy), chemotherapy alone, or BSC alone (due to poor performance status)

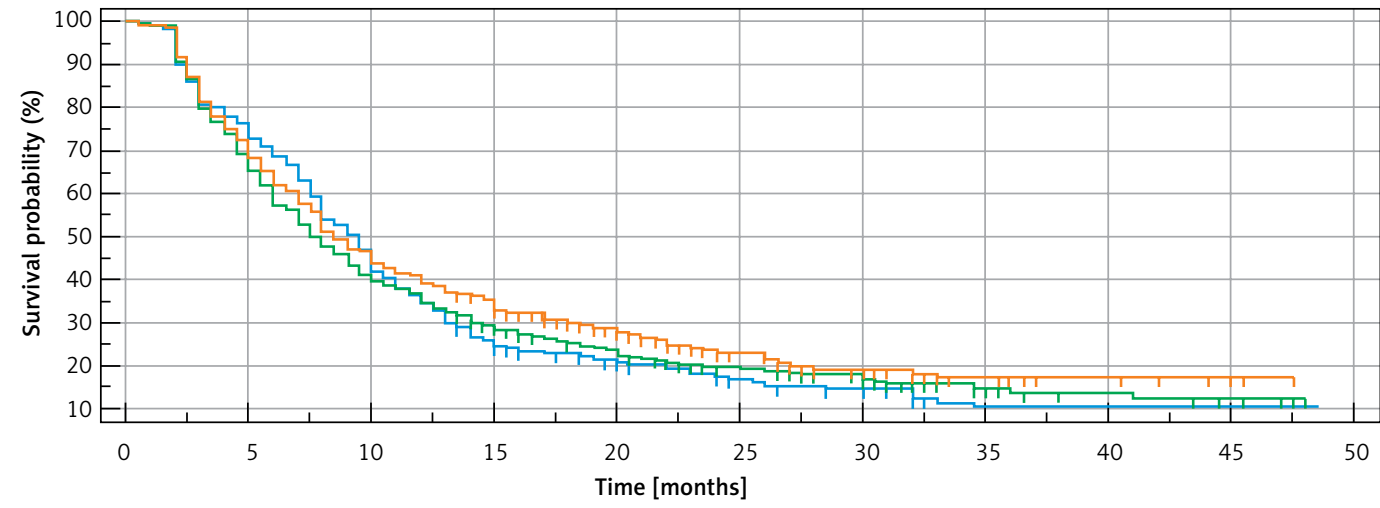

Number at risk

Group: SCLC

\begin{tabular}{|c|c|c|c|c|c|c|c|c|c|c|}
\hline 254 & 185 & 106 & 60 & 42 & 30 & 19 & 7 & 4 & 4 & 0 \\
\hline \multicolumn{11}{|c|}{ Group: non-SCC NSCLC } \\
\hline 389 & 255 & 154 & 103 & 71 & 50 & 35 & 19 & 12 & 7 & 0 \\
\hline \multicolumn{11}{|l|}{ Group: SCC } \\
\hline 354 & 242 & 155 & 110 & 70 & 43 & 24 & 15 & 9 & 3 & 0 \\
\hline Histopatnological & & & $-\mathrm{SCLC}$ & $\bar{T} \mathrm{~T}$ & $\begin{array}{l}\text { JSCLC } \\
\text { lata }\end{array}$ & $-\mathrm{sCC}$ & & & & \\
\hline
\end{tabular}

Figure 3. Survival curves of SCC, non-SCC NSCLC, and SCLC patients treated only with chemotherapy

of patients receiving chemotherapy alone with 1-year survival was significantly higher than the percentage of patients receiving modern therapies in one of the treatment lines $(p<0.000001$, $\left.\chi^{2}=77.35\right)$. Data are presented in Table I.

\section{Discussion}

Our data shows the impact of treatment methods on the overall survival of patients with locally advanced or advanced lung cancer in the conditions of actual clinical practice in Poland. The study also reveals the percentage of patients who underwent diagnosis of predictive factors and the number of patients who used personalised treatment. The study included all patients who were diagnosed with lung cancer, not just patients who fulfilled the criteria for the clinical trial. In the whole group of patients diagnosed with lung cancer, age below 65 years and being female, as well SCC or AC diagnosis, predisposed to higher median overall survival. 
Median overall survival for patients receiving immunotherapy at any of the treatment lines was 28.5 months, which was significantly higher compared to patients treated only with chemotherapy. Other studies also confirmed the significant effectiveness of immunotherapy. In the meta-analysis of seven randomised and controlled trials in advanced NSCLC, Khan et al. showed that the use of anti-PD-1 or anti-PD-L1 inhibitors improves overall survival. The risk of death during the use of immunotherapy was significantly lower compared to the use of chemotherapy $(\mathrm{HR}=0.72,95 \% \mathrm{Cl}$ : 0.63-0.82, $p<0.00001)$. However, age and sex had no impact on overall survival, but SCC diagnosis was associated with better OS [11].

It should also be remembered that the immune response is not fully explained by PD-L1 expression, and the choice of immunotherapy seems to have an advantage over chemotherapy in different groups of patients regardless of PD-L1 expression on tumour cells. The KEYNOTE-042 study proved that the efficacy of pembrolizumab in the first line of treatment could be observed in patients with locally advanced or metastatic non-small cell lung cancer without sensitising EGFR or ALK alterations and with low percentage of tumour cells with PD-L1. Patients receiving pembrolizumab, despite expression of PD-L1 on < $50 \%$ of tumour cells, had better survival compared to patients receiving chemotherapy [12]. In our study, we observed a better outcome in patients treated with pembrolizumab in the the first line ( $\geq 50 \%$ of tumour cells with PD-L1 expression) and receiving immunotherapy in the second line $(<50 \%$ of tumour cells with PD-L1 expression) compared to patients receiving chemotherapy alone.

There is a problem in the overall survival assessment in patients receiving TKIs in clinical trials because in most studies no significant improvement in OS was seen after using molecularly targeted therapies. The crossover complicates overall survival analyses in clinical trials. In randomised trials, patients initially treated with chemotherapy, after the disease progressed, received TKIs in the second or third line.

In a meta-analysis, Sellmann et al. showed that no significant differences in OS were observed in patients treated with the first and second generation of EGFR TKIs compared to patients treated with chemotherapy. The evaluation included first-line treatment and allowed method of treatment to be changed (crossover) in the case of progression. Median OS for selected clinical trials with first-line gefitinib, erlotinib, and afatinib in NSCLC patients with EGFR mutations was 17.328.2 months. Patients who received the first line of chemotherapy achieved a comparable median OS: 17.3-28.2 months. Crossover rates for patients treated with chemotherapy were $65-95 \%$.
Only results for the INFORM trial (maintenance therapy with gefitinib following platinum-based chemotherapy in EGFR mutated NSCLC patients) provided evidence that maintenance therapy with gefitinib significantly improved OS (46.9 months vs. 21.0 months, $p=0.036$ ) because of a relatively low crossover rate (53\%) [13]. The effectiveness of EGFR TKI generations older than third generation has been compared in recent clinical studies. Today, based on the results of clinical trials, it is not possible to demonstrate benefits in survival in NSCLC patients with EGFR gene mutations treated with EGFR TKIs instead of chemotherapy in firstline treatment.

Similar results were obtained for patients treated with ALK inhibitors. In a prospective study conducted by Shaw et al., crizotinib was compared with standard chemotherapy in NSCLC patients with $A L K$ gene rearrangement. During interim analysis of overall survival, there was no significant difference in overall survival between crizotinib therapy and chemotherapy $(\mathrm{HR}=1.02,95 \% \mathrm{Cl}$ : $0.68-1.54, p=0.54$ ) [14]. The ASCEND-5 study compared ceritinib with chemotherapy in NSCLC patients, who had previously progressed on crizotinib and platinum-based chemotherapy. The median OS showed no significant difference between the two groups of treatment: 23.9 months in the ceritinib group and 22.8 months in the chemotherapy group $(\mathrm{HR}=0.88,95 \% \mathrm{Cl}: 0.27-2.82)$ [15]. It has also been concluded that ALK inhibitors have a beneficial effect on overall survival in both the ceritinib group and the post-crossover chemotherapy group [16].

In our research, the use of several chemotherapy lines in patients receiving chemotherapy and the use of chemotherapy in patients receiving personalised treatment were allowed. However, the median overall survival for patients treated only with chemotherapy was 8.5 months; whereas, the median overall survival of genetically predisposed patients who received EGFR or ALK TKIs in any line of treatment was over 30 months. Unfortunately, no reliable predictors of chemotherapy have yet been developed. Attempts are being made to use such markers as ERCC1 (DNA excision repair protein), RRM1 (ribonucleoside diphosphate reductase subunit 1), TS expression (thymidylate synthase), or expression of various micro-RNAs. Some experiments have shown that patients with high expression (mRNA or protein) of ERCC1 and RRM1 are resistant to chemotherapy. Research on other markers of chemotherapy sensitivity is ongoing, such as studies on long non-coding regulatory RNAs [17].

It should also be mentioned that in our analysis, patients with SCLC received only chemotherapy, because of the lack of other therapeutic options in Poland for this period. Median overall survival 
for patients with SCLC was 6 months. Survival in SCLC patients was only slightly shorter than the survival of patients with SCC or AC. As a result, it is not pathomorphological diagnosis but the possibility of using personalised treatment that is crucial for prolonging survival in locally advanced or advanced NSCLC patients. According to the analysis of Peifer et al., small cell lung cancer has the highest mutation rate compared to other cancers (7.4 mutations per million base pairs, compared with 6.3 for melanoma and 0.4-1.5 for various other solids) [18]. Therefore, it should be hoped that soon modern immunotherapy methods will significantly improve the prognosis for patients with this type of cancer.

In conclusion, we focused on therapies that should be widely available. The use of modern therapies in one of the treatment lines compared to chemotherapy alone significantly increased the chance of long-term survival of patients with advanced lung cancer. However, not all patients who had a genetic predisposition received molecularly targeted treatment. Only 41 out of 64 patients with genetic alterations received TKIs, due to the late diagnosis of the disease. In most patients, correct and early diagnosis of lung cancer and the determination of predictive factors is required, because patients with late diagnosis have a particularly poor prognosis compared to patients treated with novel therapies.

\section{Conflict of interest}

The authors declare no conflict of interest.

\section{References}

1. Siegel RL, Miller KD, Jemal A. Cancer statistics, 2017. CA Cancer J Clin 2017; 67: 7-30.

2. Travis WD, Brambilla E, Burke AP, Marx A, Nicholson AG. Introduction to the 2015 World Health Organization classification of tumors of the lung, pleura, thymus, and heart. J Thorac Oncol 2015; 10: 1240-2.

3. Deng H, Liu J, Duan X, Liu Y. The relationship between EGFR mutation status and clinic-pathologic features in pulmonary adenocarcinoma. Pathol Res Pract 2018; 214: 450-4.

4. Gahr S, Stoehr R, Geissinger E, et al. EGFR mutational status in a large series of Caucasian European NSCLC patients: data from daily practice. Br J Cancer 2013; 109: 1821-8.

5. Dearden S, Stevens J, Wu YL, Blowers D. Mutation incidence and coincidence in non small-cell lung cancer: meta-analyses by ethnicity and histology (mutMap). Ann Oncol 2013; 24: 2371-6.

6. Soda M, Choi YL, Enomoto M, et al. Identification of the transforming EML4-ALK fusion gene in non-small-cell lung cancer. Nature 2007; 448: 561-6.

7. Rafei H, El-Bahesh E, Finianos A, Nassereddine S, Tabbara I. Immune-based therapies for non-small cell lung cancer. Anticancer Res 2017; 37: 377-87.
8. Aggarwal C, Rodriguez Abreu D, Felip E, et al. Prevalence of PD-L1 expression in patients with non-small cell lung cancer screened for enrollment in KEYNOTE-001, -010, and -024. Ann Oncol 2016; 27 (suppl 6): 1060Pa.

9. Lee CK, Man J, Lord S, et al. Checkpoint inhibitors in metastatic EGFR-mutated non-small cell lung cancer a meta-analysis. J Thorac Oncol 2017; 12: 403-7.

10. Planchard D, Popat S, Kerr K, et al. Metastatic non-smallcell lung cancer: ESMO Clinical Practice Guidelines for diagnosis, treatment and follow-up. Ann Oncol 2018; 29 (suppl 4): iv192-237.

11. Khan M, Lin J, Liao G, et al. Comparative analysis of immune checkpoint inhibitors and chemotherapy in the treatment of advanced non-small cell lung cancer: a meta-analysis of randomized controlled trials. Medicine 2018; 97: e11936.

12. Lopes G, Wu YL, Kudaba I, et al. Pembrolizumab (pembro) versus platinum-based chemotherapy (chemo) as first-line therapy for advanced/metastatic NSCLC with a PD-L1 tumor proportion score (TPS) $\geq 1 \%$ : open-label, phase 3 KEYNOTE-042 study. J Clin Oncol 2018; 36 (Suppl 18): LBA4.

13. Sellmann L, Fenchel K, Dempke WC. Improved overall survival following tyrosine kinase inhibitor treatment in advanced or metastatic non-small-cell lung cancer-the Holy Grail in cancer treatment? Transl Lung Cancer Res 2015; 4: 223-7.

14. Shaw AT, Kim DW, Nakagawa K, et al. Crizotinib versus chemotherapy in advanced ALK-positive lung cancer. N Engl J Med 2013; 368: 2385-94.

15. Kiura K, Imamura F, Kagamu H, et al. Phase 3 study of ceritinib vs chemotherapy in ALK-rearranged NSCLC patients previously treated with chemotherapy and crizotinib (ASCEND-5): Japanese subset. Jpn J Clin Oncol 2018; 48: 367-75.

16. Shaw AT, Kim TM, Crinò L, et al. Ceritinib versus chemotherapy in patients with ALK-rearranged non-small-cell lung cancer previously given chemotherapy and crizotinib (ASCEND-5): a randomised, controlled, open-label, phase 3 trial. Lancet Oncol 2017; 18: 874-86.

17. Xu R, Han Y. Long non-coding RNA FOXF1 adjacent non-coding developmental regulatory RNA inhibits growth and chemotherapy resistance in non-small cell lung cancer. Arch Med Sci 2019; 15: 1539-46.

18. Peifer M, Fernández-Cuesta L, Sos ML, et al. Integrative genome analyses identify key somatic driver mutations of small-cell lung cancer. Nat Genet 2012; 44: 1104-10. 